\title{
Hamiltonian Simple Polytopes
}

\author{
N. Prabhu
}

238 Grissom Hall, Purdue University, West Lafayette, IN 47907, LSA

prabhu@ecn.purdue.edu

\begin{abstract}
We show that for every $n>n_{0}(d)$ ( $n$ even if $d$ is odd) there exists a simple $d$-polytope with $n$ vertices, whose graph admits a Hamiltonian circuit. The result sharpens an earlier lower bound due to Victor Klee.
\end{abstract}

\section{Introduction}

Let $P$ be a simple $d$-polytope, let $h(P)$ be the length of the longest edge-cycle in $P$, and let $f_{0}(P)$ be the number of vertices in $P$. Let $S_{d}$ be the class of all simple $d$-polytopes.

In his study of paths on polyhedra [5] Klee defines the following function,

$$
K(n, d)=\max \left\{h(P) \mid P \in S_{d} ; f_{0}(P) \leq n\right\},
$$

and proves that $K(n, d) \geq(d-1)\lfloor(n-2) /(d-1)\rfloor+2$. That is, among all simple $d$-polytopes with at most $n$ vertices a polytope can be found in which the longest edge-cycle has at least $(d-1)\lfloor(n-2) /(d-1)\rfloor+2$ edges.

We show that for large enough $n\left(n \geq n_{0}(d)\right)^{1}$ Hamiltonian simple $d$-polytopes with $n$ vertices, i.e., simple polytopes whose graphs have Hamiltonian circuits, can be constructed. Our result is significant for three reasons:

1. The result closes the gap in Klee's lower bound mentioned above, by showing that, for large $n, K(n, d)=n$.

2. Equally important is the corollary of our result about the existence of simple

\footnotetext{
${ }^{1}$ Since, by definition, $d$ edges are incident at every vertex of a simple $d$-polytope, a simple $d$-polytope with $n$ vertices has $n d / 2$ edges. Therefore if $d$ is odd, $n$ cannot be odd. The above restriction is implicit in the whole discussion.
} 
$d$-polytopes with a given number of vertices. A number $n$ is said to be $d$-realizable if a simple $d$-polytope with $n$ vertices exists.

The celebrated Lower Bound Theorem [1] implies that no $n$ in the range $d+1<n<2 d$ is $d$-realizable. For $d \leq 9$, [7] lists all $n$ that are not $d$-realizable; this list shows that $n$ that are not $d$-realizable occur beyond $2 d$ as well. McMullen's conditions provide other restrictions on the $d$-realizable $n$ [3].

By constructing simple $d$-polytopes with $n$ vertices for all $n \geq n_{0}(d)$ our result shows that for each $d$ there are only finitely many $n$ that are not $d$-realizable.

3. Whether all simple polytopes of dimension higher than three have Hamiltonian circuits is still an open problem ${ }^{2}[1]$.

Hamiltonian circuits in polytopes have been a subject of continuing investigation beginning with Hamilton's observations about circuits in the dodecahedron. The existence of Hamiltonian circuits in polytopes is intimately related to the four-color problem [2]. In contrast to Kirkman's non-Hamiltonian 3-polytopes and Tutte's non-Hamiltonian simple 3-polytopes, the following classes of 3-polytopes are known to have Hamiltonian circuits [4], [6]: 3-polytopes with 4-connected graphs; simple 3 -polytopes with at most 36 vertices; simple 3-polytopes with at most two of three categories of faces: triangles, quadrilaterals, and hexagons; and simplicial 3-polytopes with maximum vertex degree six. Reference [2] contains other results and conjectures about Hamiltonian circuits in various classes of polytopes.

\section{Result}

Theorem 1. For each $d \geq 2$ a constant $n_{0}(d)$ exists such that for all $n \geq n_{0}(d) a$ Hamiltonian simple d-polytope with $n$ vertices can be constructed.

Proof. Observe that if $P$ is any Hamiltonian $d$-polytope, then a prism with basis $P$ is a Hamiltonian $(d+1)$-polytope. The prism is the convex hull of $P$ and its translate $P^{\prime}$. If $v$ is a vertex of $P$, then let $v^{\prime}$ be its neighbor in $P^{\prime}$. If $v_{0}$, $v_{1}, \ldots, v_{m}, v_{0}$ is the Hamiltonian circuit in $P$, then $v_{0}, v_{1}, \ldots, v_{m}, v_{m}^{\prime}, v_{m-1}^{\prime}, \ldots, v_{0}^{\prime}$, $v_{0}$ is a Hamiltonian circuit in the prism.

Also observe that if a simple $d$-polytope $P$ has a Hamiltonian circuit, then the $d$-polytope $P^{\prime \prime}$ obtained by truncating a vertex $v_{i}$ of $P^{3}$ also has a Hamiltonian circuit. Truncating the vertex $v_{i}$ of a simple $d$-polytope $P$ creates a new facet $F$

\footnotetext{
${ }^{2}$ Non-Hamiltonian simple 3-polytopes were discovered by Tutte [8]; non-Hamiltonian polytopes are known to exist in every dimension higher than two [2].

${ }^{3}$ Let $H$ be a hyperplane that does not contain any vertex of $P$. Further let $v_{i}$ lie in the closed half-space $H^{+}$of $H$ and the remaining vertices of $P$ in the closed half-space $H^{-}$. If $P^{n}$ is the $d$-polytope obtained by truncating the vertex $v_{i}$ in $P$, then $P^{\prime \prime}=P \cap H$ is a new facet in $P^{\prime \prime}$.
} 
which is a $(d-1)$-simplex. Each vertex of $F$ comes from an edge incident on $v_{i}$ in $P$. Let $v_{0}, \ldots, v_{i-1}, v_{i}, v_{i+1}, \ldots, v_{n}, v_{0}$ be a Hamiltonian circuit in $P$. In this circuit, replace $v_{i}$ by a Hamiltonian path in $F$, between the vertices that are adjacent to $v_{i-1}$ and $v_{i+1}$, respectively. The new circuit is Hamiltonian in $P^{\prime \prime}$.

We proceed by induction on $d$ and assuming the theorem for $d=k$ prove it for $d=k+1$.

1. Let $k+1$ be an odd number. Set $n_{0}(k+1)=2 n_{0}(k)$. Let $n \geq n_{0}(k+1)$ be an even number (see footnote 1). Then $n / 2 \geq n_{0}(k)$ and hence we have a Hamiltonian simple $k$-polytope, say $P$, with $n / 2$ vertices (inductive hypothesis). A prism with basis $P$ has $n$ vertices and is a Hamiltonian simple $(k+1)$ polytope.

2. Next assume $k+1$ is even. Since $k$ is odd, $n_{0}(k)$ is even. Inductively we have a Hamiltonian simple $k$-polytope with $2 m$ vertices for all $2 m \geq n_{0}(k)$. Thus we can construct Hamiltonian simple $(k+1)$-polytopes with $4 m$ vertices for all $4 m \geq 2 n_{0}(k)$ (by taking prisms).

Observe that truncating a vertex of a simple $(k+1)$-polytope increases the number of vertices by $k$. Further since $k$ is odd, either $k \equiv 1 \bmod 4$ (i.e., $k=4 r+1$ ) or $k \equiv 3 \bmod 4$ (i.e., $k=4 r+3$ ). If $k \equiv 1 \bmod 4$, then $2 k \equiv 2 \bmod 4$ and $3 k \equiv 3$ $\bmod 4$. On the other hand if $k \equiv 3 \bmod 4$, then $2 k \equiv 2 \bmod 4$ and $3 k \equiv 1 \bmod 4$.

Now we show that for all $n \geq 2 n_{0}(k)+4 k$ we can construct a Hamiltonian simple $(k+1)$-polytope with $n$ vertices.

Let $\left[n-\left(2 n_{0}(k)+4 k\right)\right] \equiv q \bmod k$. To construct a Hamiltonian simple $(k+1)$ polytope with $n$ vertices, it is sufficient to construct a Hamiltonian simple $(k+1)$ polytope $P$ with $2 n_{0}(k)+4 k+q$ vertices; we could then start with $P$ and truncate vertices $\left(n-\left(2 n_{0}(k)+4 k+q\right)\right) / k$ times to obtain a Hamiltonian simple $(k+1)$ polytope with $n$ vertices (each truncation preserves Hamiltonicity and increases the vertex-count by $k$ ).

Assume $k \equiv 1 \bmod 4$. Let $2 n_{0}(k)+4 k+q-k \equiv r \bmod 4$. If $r=0$, then starting with a Hamiltonian simple $(k+1)$-polytope with $2 n_{0}(k)+3 k+q$ vertices and truncating a vertex we get a Hamiltonian simple polytope with $2 n_{0}(k)+$ $4 k+q$ vertices. If $r=1$ we start with a Hamiltonian simple $(k+1)$-polytope with $2 n_{0}(k)+2 k+q$ vertices and truncate two vertices to get a Hamiltonian simple $(k+1)$-polytope with $2 n_{0}(k)+4 k+q$ vertices. If $r=2$ we start with a Hamiltonian simple $(k+1)$-polytope with $2 n_{0}(k)+k+q$ vertices and truncate three vertices. Finally if $r=3$ we start with a Hamiltonian simple $(k+1)$-polytope with $2 n_{0}(k)+q$ vertices and truncate four vertices to obtain a Hamiltonian simple $(k+1)$-polytope with $2 n_{0}(k)+4 k+q$ vertices.

The arguments are similar if $k \equiv 3 \bmod 4$ except that if $2 n_{0}(k)+4 k+q-k \equiv r$ $\bmod 4$, for $r=0,1,2,3$ we start with Hamiltonian simple $(k+1)$-polytope with $2 n_{0}(k)+3 k+q, 2 n_{0}(k)+q, 2 n_{0}(k)+k+q, 2 n_{0}(k)+2 k+q$ vertices, respectively, and truncating one, four, three, or two times respectively.

The above argument shows that a Hamiltonian simple $(k+1)$-polytope with $n$ vertices can be constructed for all $n \geq 2 n_{0}(k)+4 k$. 
In the above construction

$$
n_{0}(k+1)= \begin{cases}2 n_{0}(k) & \text { if } k+1 \text { is odd } \\ 2 n_{0}(k)+4 k & \text { if } k+1 \text { is even }\end{cases}
$$

Solving the recurrence equation with $n_{0}(1)=2$ we see that $n_{0}(k)=O\left(k 2^{k}\right)$.

\section{Remark}

It is not known if a Hamiltonian simple $d$-polytope with $n$ vertices can be found for every $n$ that is $d$-realizable.

\section{References}

1. Barnette, D. W., A proof of the lower bound conjecture for convex polytopes, Pacific J. Math. 46 (1973), 349-354.

2. Barnette, D., and Rosenfeld, M., Hamiltonian circuits in certain prisms, Discrete Math. 5 (1973), 389-394.

3. Brøndsted, A., An Introduction to Convex Polytopes, Springer-Verlag, New York, 1983.

4. Grünbaum, B., Convex Polytopes, Wiley Interscience, New York, 1966.

5. Klee, V., Paths on polyhedra, II, Pacific J. Math., 17(2) (1966), 249-262.

6. Klee, V., and Kleinschmidt, P., Convex polytopes and related complexes, in Handbook of Combinatorics, North-Holland, Amsterdam, 1994, to appear.

7. Lee, C. W., Counting the Faces of Simplicial Convex Polytopes, Ph.D. Thesis, Cornell University, 1981.

8. Tutte, W. T., On Hamiltonian circuits, J. London Math. Soc., 21 (1946), 98-101.

Received December 6, 1993, and in revised form February 1, 1995. 